\title{
Evidences that Intake of L-valine May Affect the Lifespan-specific Local Gene Network Pattern in Caenorhabditis Elegans
}

\author{
Hong-yuan WANG ${ }^{1,2}$ and Zhi-zhou ZHANG ${ }^{1,2, a,{ }^{*}}$ \\ ${ }^{1}$ School of Chemistry and Chemical Engineering, Harbin Institute of Technology, Harbin \\ 150001, China \\ ${ }^{2}$ School of Marine Science and Technology, Harbin Institute of Technology (Weihai), \\ Weihai 264209, China \\ azhangzzbiox@hitwh.edu.cn \\ ${ }^{*}$ Corresponding author
}

Keywords: L-valine, Longevity, Local gene network, Pattern, C. elegans.

\begin{abstract}
The purpose of this study is to preliminarily investigate whether branched-chain amino acids (BCAAs) prolong the lifespan of model organism Caenorhabditis elegans $(C$. elegans) through modifying the ageing-related local gene network (LGN) pattern. After synchronization adult $C$. elegans were fed with living $E$. coli strain OP50 on nematode growth medium (NGM) agar plates with or without BCAAs (L-valine, L-leucine, L-isoleucine). L-valine extended the average lifespan of $C$. elegans significantly at low concentration $(0.1 \times)$, but the other two amino acids affected lifespan slightly. Quantitative polymerase chain reaction (QPCR) was employed to quantify expression levels of thirteen genes that are closely related with BCAAs metabolic modules in the LGN. It was found that the thirteen genes' expression levels were differentially affected (some up-regulated, some down-regulated, and some had over 10-fold changes in the expression level) by intake of $0.1 \times \mathrm{Val}$, suggesting that the extension of lifespan through $0.1 \times \mathrm{V}$ al was possibly through some uncharacterized lifespan-specific local gene network pattern change resulted from differential expression-level changes of a group of genes.
\end{abstract}

\section{Introduction}

C. elegans has been a good model organism for lifespan studies [1]. Various compounds were demonstrated to be strongly linked to longevity in different model organisms. In 2007, Bass TM et al testified resveratrol in some trials caused slight increases in lifespan in $C$. elegans in both wild-type and sir-2.1 mutant populations [2,3]. Later on in 2008, Kimberley Evason et al indicated valproic acid could not only be in favor of life extension, but also delay age-related degeneration of physiologic processes that mediate body movement [4]. Notably in 2012 Paola V et al reported that very low concentration $(1 \mathrm{mM})$ of ethanol could improve the starvation survival of L1 larvae [5]. In the same year Kim Zarse et al found L-theanine extended C. elegans lifespan at a wide range of concentration from $100 \mathrm{nM}$ to 10 micromolar [6]. These results demonstrated that small chemicals or nutrients can modulate ageing-related metabolism equilibrium or regulate some ageing-related signaling pathways in C. elegans.

Recent intriguing reports indicated that BCAA-enriched mixture consisting of L-valine, L-isoleucine, and L-leucine could steadily increase the average lifespan of mice [7]. This was likely the consequence of increased mitochondrial biogenesis and reduced oxidative stress in cardiac and skeletal muscles via eNOS-mediated mechanisms [7]. The increased survival 
with BCAAs in Saccharomyces cerevisiae was also reported in 2009 by Alvers A.L et al [8]. But so far the role of BCAAs in affecting lifespan in the model organism C. elegans has not been thoroughly studied.

In this report we tested the impact of L-valine, L-leucine and L-isoleucine on the lifespan of $C$. elegans at different concentrations, and then measured the expression levels of thirteen genes that are closely linked with BCAAs metabolic modules. Our results provided some new evidence that the extension of lifespan through $0.1 \times \mathrm{Val}$ was possibly through lifespan-specific local gene network pattern change resulted from differential expression-level changes of a dozen of genes.

\section{Methods}

\section{Compounds}

L-valine, L-leucine, and L-isoleucine were obtained from Sangon (Shanghai). For each amino acid, the final concentrations in NGM agar plates of $(1 \times)$ were $C_{V a l(1 \times)}=0.649 \mathrm{mM}$, $\mathrm{C}_{\text {Leu(1x) }}=2.88 \mathrm{mM}$, and $\mathrm{C}_{\operatorname{lle}(1 \times)}=0.577 \mathrm{mM}$.

\section{Ageing Related Local Gene Network}

Construction of ageing-related local gene network was started with 18 genes with known functional association with lifespan determination in $C$ elegans. WormNet provided a tool to construct functional connections among a group of genes. The 18 lifespan-related genes were found to link total 200 genes. All these 218 genes (not shown) were then employed to construct local gene network in KEGG database using KGML software [9-11], but only 16 genes were able to be linked with each other as LGN within known biological pathways. Interestingly, the constructed LGN was well connected with amino acids metabolic modules. In the LGN, there are about 13 genes have distinct closeness with the metabolic modules of branched-chain amino acids (Fig. 1 and Table 1, all related information can be checked in KEGG database).

\section{Maintenance and Cultivation of Nematodes}

The $C$. elegans strain for the experiment was wild-type N2. All experiments operations related with nematodes were performed exactly as the literatures previously described [12-15]. The C. elegans were cultured onto NGM agar plates at $22^{\circ} \mathrm{C}$ and fresh living E. coli OP50 bacteria were used as the only food source.

\section{Lifespan Assay}

After synchronization L4 larvae were cultured on NGM agar plates (with and without L-valine, L-leucine and L-isoleucine). The beginning of $C$. elegans hatching was recorded as the day zero, and then nematodes were transferred daily to fresh plates to until all died. The survival and death of nematodes were cultivated on different plates was recorded in detail every day. SPSS Statistics 19.0 software was used for statistical analysis after the end of experiments. 


\section{Motility}

We divided motility into four classes, where "4" animals move spontaneously and smoothly, leaving sinusoidal and symmetric tracks; "3" animals move spontaneously at lower speed; "2" animals only move slowly when prodded with a platinum wire; " 1 " animals only move the mouth or tail. Worms after adulthood were observed and recorded daily.

Table 1. QPCR primers for 13 genes that are closely related with BCAAs metabolism in LGN

\begin{tabular}{|c|c|c|c|}
\hline No. & Gene name & Primer & Primer sequence(5'-3') \\
\hline 1 & alh-9 & $\mathrm{q} 1-\mathrm{q} 2$ & $\begin{array}{l}\text { 5'-GCTCCAGCAAATAACTCCCC-3' } \\
\text { 5'-TGTTCAAATCAGCATCCTCGT-3', }\end{array}$ \\
\hline 2 & sptl-2 & $q 3-q 4$ & $\begin{array}{l}\text { 5'-CTGGAATGTGCTTGGAACC-3' } \\
\text { 5'-TTGTGCTCAAATACTTTTGTAGAA-3, }\end{array}$ \\
\hline 3 & cyp-33E1 & q5-q6 & $\begin{array}{l}\text { 5'-AAATGTTCCGTAAGCAGGC-3' } \\
\text { 5'-TTCATAGTAACTCCGACTTCCTT-3' }\end{array}$ \\
\hline 4 & unc- 25 & $\mathrm{q} 7-\mathrm{q} 8$ & $\begin{array}{l}\text { 5'-GGGAAGCAGTTGGATGGG-3' } \\
\text { 5'-TTCTCCTGGTAAAATAGTTGGC-3' }\end{array}$ \\
\hline 5 & cts-1 & q9-q10 & $\begin{array}{l}\text { 5'-GGTAGCACCGTAGTTCAAAA-3' } \\
\text { 5'-CGGAGAAGGAAAGGTATGGAT-3' }\end{array}$ \\
\hline 6 & $\mathrm{C} 14 \mathrm{E} 2.2$ & q11-q12 & $\begin{array}{l}\text { 5'-AGGAGACCACTTTCCAGGCT-3' } \\
\text { 5'-CGAACGAGGTGATCGACTTG-3' }\end{array}$ \\
\hline 7 & C32F10.8 & q13-q14 & $\begin{array}{l}\text { 5'-AAGATGGAGTATGCTGTTCGTG-3' } \\
\text { 5'-ATTGCGATCCTTGAGCGTAG-3' }\end{array}$ \\
\hline 8 & odc-1 & q17-q18 & $\begin{array}{l}\text { 5'-TGTATCTGACCCAACTGCTACG-3' } \\
\text { 5'-TCTCCCACATTTCCTCGCTC-3' }\end{array}$ \\
\hline 9 & $\mathrm{C} 05 \mathrm{D} 2.3$ & $\mathrm{q} 19-\mathrm{q} 20$ & $\begin{array}{l}\text { 5'-TACTGGGATGGAATAAGGACC-3' } \\
\text { 5'-GCTCTATAGCGTCGATCAAAAT-3' }\end{array}$ \\
\hline 10 & vrs-2 & $\mathrm{q} 21-\mathrm{q} 22$ & $\begin{array}{l}\text { 5'-CTGCTGAAATGGGATGAGGA-3' } \\
\text { 5'-GTGGTTGCCTGAGTGAAGTTAT-3' }\end{array}$ \\
\hline 11 & ctl-3 & q23-q24 & $\begin{array}{l}\text { 5'-AGACACCACTTCTCGTTCGTT-3' } \\
\text { 5'-GGTAATGCGTGTCCGTGTAG-3' }\end{array}$ \\
\hline 12 & C28h8.11 & $\mathrm{q} 25-\mathrm{q} 26$ & $\begin{array}{l}\text { 5'-CGTGTCCGTATTTGGGTTCT-3' } \\
\text { 5'-AAGCATCCGCAGTTTTATGA-3' }\end{array}$ \\
\hline \multirow[t]{2}{*}{13} & Т22H6.2 & $\mathrm{q} 27-\mathrm{q} 28$ & $\begin{array}{l}\text { 5'-GGTAGCCTAATGGTTATTTTCG-3' } \\
\text { 5'-CACGCACTGTCTACGTGTTTC-3' }\end{array}$ \\
\hline & GAPDH & & $\begin{array}{l}\text { 5'-ATGCCAAAGCCAAGTGTCG-3' } \\
\text { 5'-TGTTGGAGTATCCGAACTCGTTAT-3' }\end{array}$ \\
\hline
\end{tabular}

\section{Detection of Gene Expression}

Total RNA was extracted from nematodes fed amino acids extending lifespan significantly using UNIQ-10 column Trizol total RNA extraction kit (Sangon, Shanghai) and was reverse transcribed into cDNA using AMV first strand cDNA synthesis kit (Sangon). Thirteen genes that are closely related with BCAAs metabolic modules in the LGN were selected for real-time quantitative PCR (QPCR) analysis. Primers were listed in Table 1. QPCR with a final volume of $12 \mathrm{uL}$ contained $6 \mathrm{uL} 2 \times \mathrm{NPK} 62 \mathrm{QPCR}$ buffer (GREDBIO), 0.2uL cDNA, 1.5uL primer pair, 4.3uL ddH2O was performed on QPCR machine TP810 R-1447 (Takara). QPCR reaction began with a denaturation step at $94^{\circ} \mathrm{C}$ for $2 \mathrm{~min}$, followed by 65 cycles of amplification at $94^{\circ} \mathrm{C}$ for $30 \mathrm{~s}, 60^{\circ} \mathrm{C}$ for $1 \mathrm{~min}$ and $72^{\circ} \mathrm{C}$ for $1 \mathrm{~min}$. PCR ended with $72^{\circ} \mathrm{C}$ for $5 \mathrm{~min}$. At the end of the amplification, a melting analysis was performed. 


\section{Results and Discussion}

\section{Construction of Aging-related Local Gene Network of C. elegans}

A local gene network was constructed that included both known ageing-related genes (such as age-1, akt-1 and pdk-1) and BCAAs metabolic modules. Especially, L-valine (C00183) is located directly in the LGN. Thirteen genes (Table 1) in the LGN that have been rarely studied were selected for gene expression measurement since they are very close to BCAAs metabolic modules.

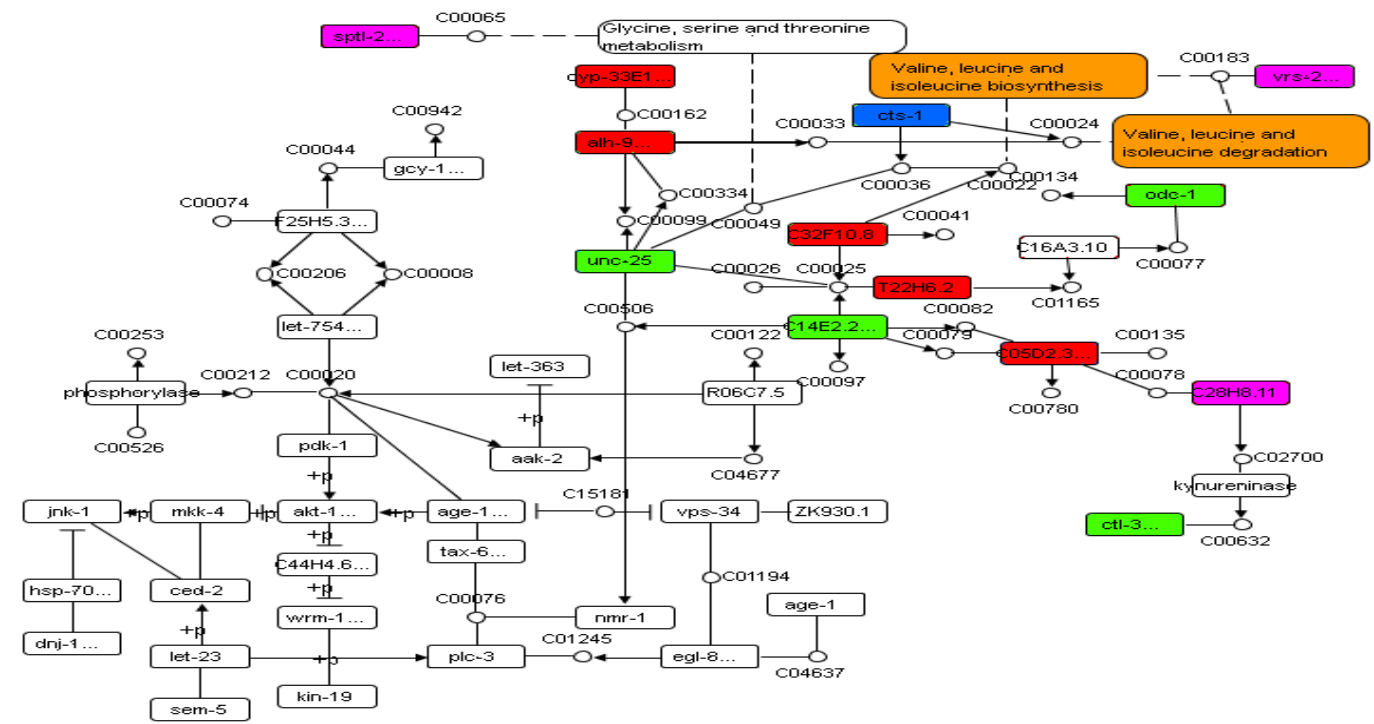

Figure 1. Ageing-related local gene network of C. elegans generated with KGML tools. Possible lifespan-related genes and metabolic modules are connected with each other by biochemical pathways. Small chemicals related with metabolic modules or signaling pathways were labeled by circle. Colored boxes represent the 13 genes that are close to BCAAs metabolic modules and subjected to qPCR analysis. Red: up-regulated then down-regulated;

Green: down-regulated then up-regulated; Pink: up-regulated; Blue: down-regulated.

\section{BCAAs Affect Lifespan in C. elegans}

Applying L-valine, L-leucine, L-isoleucine separately at three different concentrations $(0.1 \times$, $1 \times$ and $10 \times$ ) to $C$. elegans using the above-mentioned methods affects lifespan (Table 2). The results showed that $0.1 \times$ Val could extend the average lifespan significantly $(\mathrm{P}=0.001<0.01)$, and also increase the physiological activity (crawling speed, Fig. 2) while Leu and Ile had no significant impact on $C$. elegans survival.

\section{L-valine Influence the Expression of Aging-related Genes}

RNA was extracted from nematodes fed with $0.1 \times$ Val after $4 d, 8 d$ and $12 d$, then the expression of lifespan-related genes in LGN was detected by QPCR. We used a comparative method $\left(2^{-\Delta \Delta C t}\right)$ for quantitative statistics analysis and regarded GAPDH as reference gene with the prerequisite that all primer pairs had similar amplification efficiency (close to 1.0). Fig.3 demonstrated QPCR results for 13 selected genes. Five genes (alh-9, cyp-33E1, C32f10.8, C05D2.3 and T22H6.2, red-colored in Fig1) were first up-regulated then down-regulated; Four genes (unc-25, C14E2.2, ctl-3 and odc-1, green-colored in Fig1) were down-regulated first then up-regulated; Three genes (sptl-2, vrs-2 and C28h8.11, colored pink 
in Fig1) were differentially up-regulated while one gene (cts-1,colored blue in Fig1) was down-regulated.
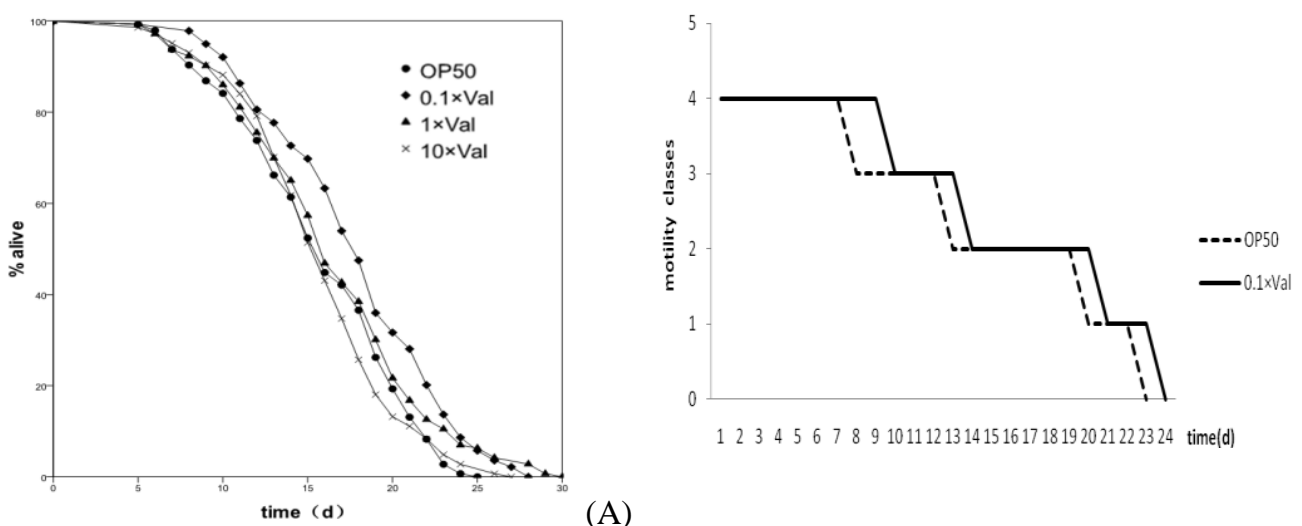

(A)

(B)

Figure 2. The low concentration of L-valine affected the lifespan and motility of adult C. elegans.

(A)Kaplan-Meier survival curves. (B) Motility assay results for $0.1 \times \mathrm{L}$-valine.

Table 2. Summary table of lifespan assays $(* * \mathrm{P}<0.01)$

\begin{tabular}{|c|c|c|c|c|}
\hline Treatment & $\begin{array}{c}\text { Average lifespan (d) } \\
( \pm \text { SD) }\end{array}$ & $\begin{array}{c}\text { P value } \\
(\text { vs. OP50) }\end{array}$ & $\begin{array}{c}\text { Number of } \\
\text { experiments }\end{array}$ & $\begin{array}{c}\text { Number of } \\
\text { nematodes }\end{array}$ \\
\hline Control & $15.786 \pm 0.406$ & -- & 3 & 145 \\
\hline $0.1 \times$ Val & $17.842 \pm 0.428$ & $0.001 * *$ & 3 & 139 \\
\hline $1 \times$ Val & $16.524 \pm 0.458$ & 0.228 & 3 & 143 \\
\hline $10 \times$ Val & $15.743 \pm 0.379$ & 0.938 & 3 & 144 \\
\hline $0.1 \times$ Leu & $15.156 \pm 0.419$ & 0.280 & 3 & 141 \\
\hline $1 \times$ Leu & $15.464 \pm 0.438$ & 0.590 & 3 & 140 \\
\hline $10 \times$ Leu & $16.313 \pm 0.484$ & 0.402 & 3 & 134 \\
\hline $0.1 \times$ Ile & $15.336 \pm 0.512$ & 0.488 & 3 & 134 \\
\hline $1 \times$ Ile & $15.979 \pm 0.412$ & 0.740 & 3 & 140 \\
\hline $10 \times$ Ile & $15.216 \pm 0.484$ & 0.366 & 3 & 139 \\
\hline
\end{tabular}

\section{Summary}

Results in this study indicated that L-valine significantly extends the average lifespan of C. elegans at low concentration $(0.1 \times)$. Besides, the metabolic modules of branched-chain amino acids are directly associated with the ageing-related local gene network, providing a convenience that LGN can be employed as a platform to investigate molecular mechanisms of lifespan determination.

BCAAs (L-valine, L-isoleucine, and L-leucine) has the function of extending lifespan of mice because of increased mitochondrial biogenesis and reduced oxidative stress in cardiac and skeletal muscles via eNOS-mediated mechanisms [7]. Reports indicated most long-lived mutants containing mitochondrial ETC mutants, IIS mutants, and translation mutants all have increased levels of BCAAs in $C$. elegans $[12,13]$. TOR pathway mutants, dietary restriction mutants and other translation mutants were also reported to have an under-appreciated function of amino-acid metabolism in longevity [14].

L-valine directly appears in the lifespan-related LGN, and participates in several signaling pathways such as valine-leucine-isoleucine degradation, valine-leucine-isoleucine biosynthesis, 
penicillin and cephalosporin biosynthesis, cyanoamino acid metabolism and so on. What is the role of these signaling pathways for ageing regulation or lifespan determination is unknown.
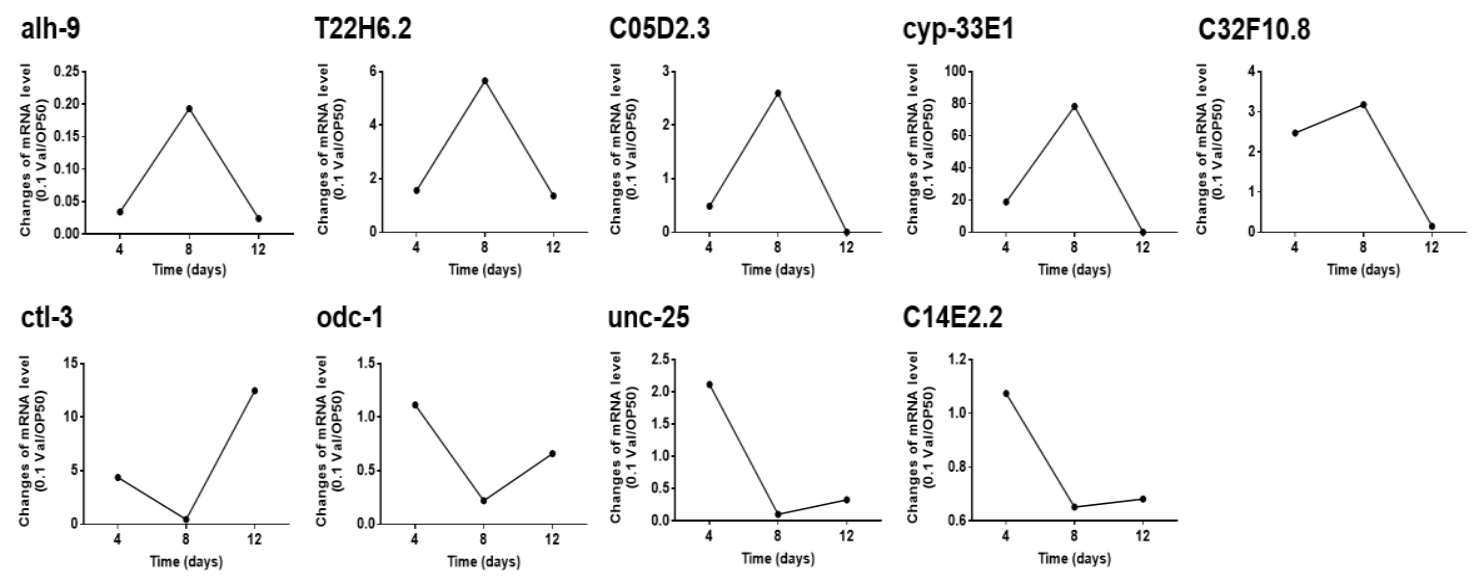

\section{C14E2.2}
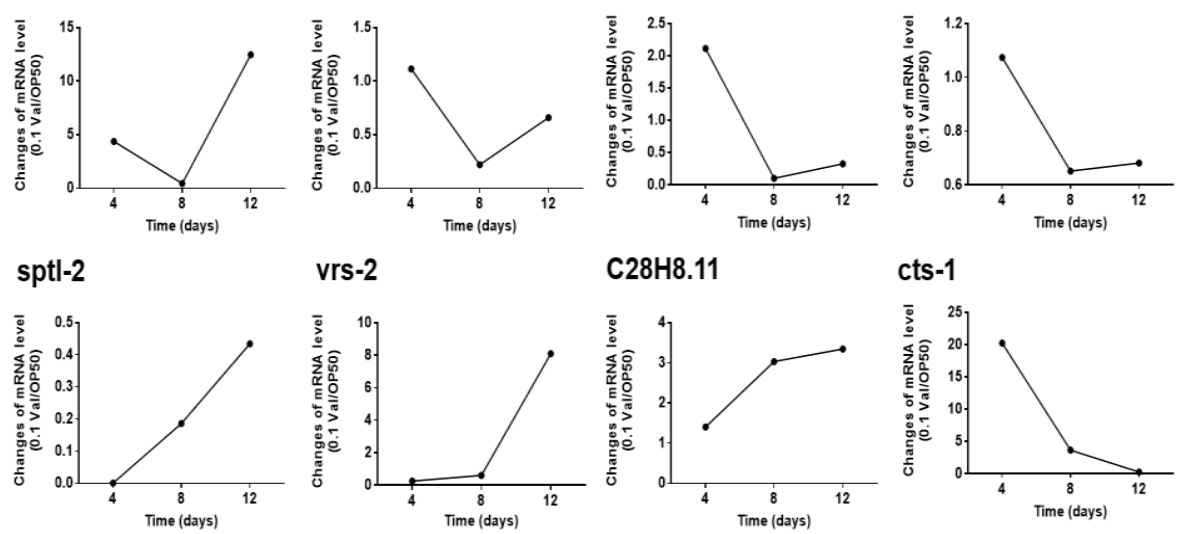

Figure 3. QPCR results of expression level changes for 13 genes closely linked with BCAAs metabolic modules. Also see labels in Figure.1.

Thirteen selected genes that are closely linked with BCAAs metabolic modules in the LGN were subjected to gene expression quantification in this study, while the LGN has at least 39 genes. The thirteen genes didn't include any one of the 18 LGN-construction-starting genes with known lifespan-related biological functions (See Methods). There are several genes such as let-363, pdk-1 and akt-1 that are involved in longevity-related mTOR signaling pathway. Besides, let-363 encodes a rapamycin complex-associated protein. Next step is to systematically investigate the gene expression states in the larger local gene network.

One potentially important aspect is molecular network equilibrium theory. In this theory, different metabolic equilibrium states have different logistics distribution and lead to different network patterns or physiological phenotypes. Local gene network is actually a local molecular network in which proteins, enzymes, nutrients, metabolites and their subcellular localizations are key factors that determine the equilibrium states of local molecular network. Theoretically, expression-level changes in some genes in the LGN can lead to significant metabolic trend change or network pattern change. Intake of L-valine may change the pattern of ageing-related local molecular network, and this point of view also needs future detailed experimental evaluation.

\section{Acknowledgement}

This work was funded by NSFC (No.31071170), GREDBIO fund, HIT (hitwh200904), 985 Fund, HIT-NSRIF (2011101) and The Science and Technology Development Projects of Weihai (2011DXGJ13, 2012DXGJ02). 


\section{References}

[1] Gershon H, et al., Caenorhabditis elegans-a paradigm for aging research: advantages and limitations, Mechanisms of Ageing and Development. 123 (2002) 261-274.

[2] Bass TM, et al., Effects of resveratrol on lifespan in Drosophila melanogaster and Caenorhabditis elegans, Mech Ageing Dev. 128 (2007) 546-552.

[3] Zarse K, et al., Differential effects of resveratrol and SRT1720 on lifespan of adult Caenorhabditis elegans, Horm Metab Res. 42 (2010) 837-839.

[4] Evason K, et al., Valproic acid extends Caenorhabditis elegans lifespan, Aging Cell. 7 (2008) 305-317.

[5] Castro PV, et al., Caenorhabditis elegans battling starvation stress: low levels of ethanol prolong lifespan in L1 larvae, PLoS ONE. 7 (2012) 1-11.

[6] Zarse K, Jabin S, Ristow M, L-Theanine extends lifespan of adult Caenorhabditis elegans, Eur J Nutr. 51 (2012) 765-768.

[7] D'Antona G, et al., Branched-chain amino acid supplementation promotes survival and supports cardiac and skeletal muscle mitochondrial biogenesis in middle-aged mice, Cell Metabolism. 12 (2010) 362-372.

[8] Alvers AL, et al., Autophagy and amino acid homeost asis are required for chronological longevity in Saccharomyces cerevisiae, Aging Cell. 8 (2009) 353-369.

[9] Zhang Z, et al., Local gene network pattern (LGNP) approach to tackle mechanisms of complicated phenotypes, The 2nd International Conference on Bioinformatics and Biomedical Engineering. (2008) May 16-May 18.

[10] Zhang T, et al., Molecular association between diabetes-specific local gene network and nutrient metabolism modules, The 4th International Conference on Bioinformatics and Biomedical Engineering. (2010) June 16-June 20.

[11] Tan XQ, et al., Using local gene network approach to functionally link lifespan genes and nutrient metabolism modules in C. elegans, International Conference on Remote Sensing, Environment and Transportation Engineering. (2011) June 24-June 26.

[12] Fuchs S, et al., A metabolic signature of long life in Caenorhabditis elegans, BMC Biol. 8 (2010) 14.

[13] Falk M, et al., Metabolic pathway profiling of mitochondrial respiratory chain mutants in C. elegans, Mol Genet Metab. 93 (2008) 388-397.

[14] Gallo M, Riddle DL, Regulation of metabolism in Caenorhabditis elegans longevity, J Biol. 9 (2010)7. 